\title{
Study on the Preparation of Sm Doped TiO2 Catalyst by Microwave Hydrothermal Method and its Photocatalytic Activity
}

\author{
Yuejie $\mathrm{Lu}^{\mathrm{a}}$, Xin Wang ${ }^{\mathrm{b}}$ and Xianjun $\mathrm{Bi}^{\mathrm{C}_{*}}$ \\ Institute of Chemistry and Chemical Engineering, Yunnan Normal University, Kunming 650500, \\ Yunnan, P. R. China \\ a1079260276@qq.com, b965184394@qq.com, 9bixj159@aliyun.com \\ *The corresponding Author
}

\begin{abstract}
Keywords: Microwave hydrothermal method; Samarium doped; TiO2 photocatalyst; Microwave enhanced; Photocatalytic activity
\end{abstract}

\begin{abstract}
Samarium doped $\mathrm{TiO} 2$ photocatalyst $\mathrm{TiO} 2-\mathrm{Sm}$ was prepared by microwave hydrothermal method using Samarium nitrate and tetrabutyl titanate as raw materials. Degradation of methyl orange was used to photocatalytic reaction of $\mathrm{TiO} 2-\mathrm{Sm}$ respectively under the conditions of visible light, microwave (MW), ultraviolet (UV), ultraviolet microwave (UV-MW) and ultraviolet microwave ultrasonic (UV-MW-UT). The TiO2-Ag was characterized by SEM, TG-DSC-DTG, IR and UV-Vis. The results showed that the optimal conditions of TiO2-Sm prepared by microwave hydrothermal method was $\mathrm{n}(\mathrm{Sm} 3+) / \mathrm{n}(\mathrm{Ti} 4+)=0.003$, microwave power $450 \mathrm{~W}$, reacting time for $3 \mathrm{~h}$ at $170^{\circ} \mathrm{C}$ and calcining time for $3 \mathrm{~h}$ at $700^{\circ} \mathrm{C}$. The degradation rate was $11.51 \%, 96.83 \%, 99.5 \%$ and $100 \%$ respectively by MW, UV, UV-MW and UV-MW-UT for 35 min degradation. Sm doped had great influence on the crystal formation which widen the temperature range of catalyst from amorphous to anatase and suppressing the anatase structure into rutile structure. Moreover Sm doped produced more hydroxyl groups and could also widen the absorb range of ultraviolet and visible light which caused the enhancement of the photocatalytic activity of TiO2-Sm.
\end{abstract}

\section{Introduction}

Water being the source of life, it cannot be replaced in any case, and human beings cannot survive without water environment and development since ancient times. As a result of all kinds of life and production of human beings, the pollution of water sources is serious. Nowadays, people are aware of the importance of environmental pollution control and take measures to control water pollution.

In recent decades, the development of new green semiconductor materials become researchers darling. TiO2 has the advantages of low cost, good chemical stability, non-toxic, no two pollution, so it is widely used[1,2]. Photocatalytic technology is easy to operate and control, and it's good effect and no two pollution in the process of environmental pollution treatment[3]. However, the response of $\mathrm{TiO} 2$ photocatalyst to visible light is not good, which restricts its application in practical production and life[4]. In the aspect of ion doping, there have been a lot of in-depth studies. La3+, $\mathrm{Ce} 3+, \mathrm{Er} 3+, \mathrm{Pr} 3+, \mathrm{Gd} 3+, \mathrm{Nd} 3+$ or $\mathrm{Sm} 3+$ doped $\mathrm{TiO} 2$ photocatalysts have been prepared by sol-gel method[5]. Recently, the microwave hydrothermal method has been applied to the rare earth metal doped $\mathrm{TiO} 2$ to obtain better photocatalytic effect[6]. In this paper, Sm doped $\mathrm{TiO} 2$ photocatalyst prepared by microwave hydrothermal method, and the effects of various auxiliary conditions on the photocatalytic activity of $\mathrm{TiO} 2-\mathrm{Sm}$ catalyst were investigated. Through the characterization and photocatalytic degradation reaction results of analysis so as to further improve the photocatalytic activity of $\mathrm{TiO} 2$ catalyst. 


\section{Experimental Section}

Experimental Reagents and Instruments. The Reagent: Samarium nitrate (AR), butyl titanate (AR), ethanol (AR), nitric acid, methyl orange, ultra-pure water.

Instrument: $\mathrm{XH}-800 \mathrm{~S}$ microwave hydrothermal parallel synthesis and XH-300UL synthetic instrument computer microwave ultrasonic wave combined catalytic (Beijing Xianghu science and Technology Development company), DZF-6030A type vacuum drying oven (Shanghai Hengke Technology company), GGZ2300Z a homemade photocatalytic device of type high pressure mercury lamp (Shanghai Yaming Electrical Appliance company), HC-2064 high speed centrifuge(innovative Limited company by Zhongjia branch company), XL30ESEM-TMP scanning electron diffraction microscope (Holland), STA 449 F3 thermal analyzer (Netzsch), Ten Sor27 infrared spectroscopy (Bruke, Germany), TU-1901 UV-VIS spectrophotometer (Beijing spectrum analysis), UV-2550 ultraviolet spectrophotometer (Shimadzu, Japan).

Preparation of TiO2 -Sm Catalyst. Adding $17.5 \mathrm{~mL}$ ethanol into beaker after taking $3.4 \mathrm{~mL}$ tetrabutylorthotitanate, and stirring to form A solution. In different material $n\left(\mathrm{Sm}^{3+}\right) / \mathrm{n}\left(\mathrm{Ti}^{4+}\right)$ of silver nitrate dissolved in ultra-pure water to obtain B solution. the B solution was added drop wise into A solution, and switched to PTFE high pressure reactor, then changing the microwave hydrothermal parallel synthesis of microwave power, hydrothermal reaction temperature and reaction time. Reaction, filtration, ultra pure water washing made white filter cake. Finally, the sample fully grinded after the vacuum drying oven dried for $3 \mathrm{~h}$. The $\mathrm{Sm}$ doped $\mathrm{TiO}_{2}$ photocatalyst $\mathrm{TiO}_{2}-\mathrm{Sm}$ was prepared in a high temperature box type resistance furnace at different temperatures and placed in dryer.

Photocatalytic Activity Test. The photocatalytic activity of $\mathrm{TiO}_{2}-\mathrm{Sm}$ catalyst was tested with refer to literature ${ }^{[7-9]}$. By changing the reaction time to do photocatalytic reaction respectively under the degradation conditions for UV, MW, UV-MW and UT-UV-MW in computer combined of microwave and ultrasonic catalysis synthesis instrument. After taking the supernatant liquid, The determination of removal efficiency of $\mathrm{TiO}_{2}-\mathrm{Ag}$ was synthesized by microwave hydrothermal method. With the degradation rate of formula calculated the degradation rate of methyl orange: $\eta=$ $\left(\mathrm{A}_{0}-\mathrm{A}\right) / \mathrm{A}_{0} \times 100 \%\left(\mathrm{~A}_{0}\right.$ and $\mathrm{A}$ respectively before and after the absorbance of methyl orange degradation).

The visible light photocatalytic activity test: First, the methyl orange solution put into the beaker, adding a certain amount of catalyst. Secondly, every half hour sampling at 12:00 to 4:00 time after the sun light irradiation in the suitable place of the campus of the Yunnan Normal University of the Chenggong District of Kunming City. Finally, according to the above analysis steps to calculate the degradation rate of methyl orange. Blank experiments showed that not only the catalyst in the absence of illumination, but also in the presence of catalysts without light, the concentration of methyl orange solution unchanged.

\section{Results and Discussion}

Photocatalytic Property.1) Effect of Sm doped on photocatalytic activity.

Fig. 1 was effect of the change amount of samarium nitrate on $\mathrm{TiO}_{2}-\mathrm{Sm}$ photocatalytic activity in UV irradiation degradation of $50 \mathrm{~min}$. The preparation of $\mathrm{TiO}_{2}-\mathrm{Sm}$ catalyst by microwave hydrothermal method in the condition of microwave power $450 \mathrm{~W}$, the reaction temperature $170{ }^{\circ} \mathrm{C}$, the reaction time $3 \mathrm{~h}$, calcination temperature $700{ }^{\circ} \mathrm{C}$ and the calcination time $3 \mathrm{~h}$. When the amount of proper doped $\mathrm{Sm}^{3+}$, doped ions becomed the center separation of electrons and holes, thus the photocatalytic activity of the catalysts was improved. when the doping ions were too large, the distance between the doped ions was too small, which made it become the recombination center of electron and hole, thus decreased the photocatalytic activity of catalysts ${ }^{[10,11]}$. The result of the degradation rate first increased and then decreased with the increase of Sm doping amount could be seen from Fig. 1. And more the degradation rate reached a maximum value of $97.80 \%$ when $\mathrm{Sm}$ 
doping amount $\mathrm{n}\left(\mathrm{Sm}^{3+}\right) / \mathrm{n}\left(\mathrm{Ti}^{4+}\right)$ was $0.3 \%$, which indicated that the percentage of optimal amount of doped material was $0.3 \%$ and $\mathrm{TiO}_{2}-\mathrm{Sm}$ photocatalyst had high photocatalytic activity.

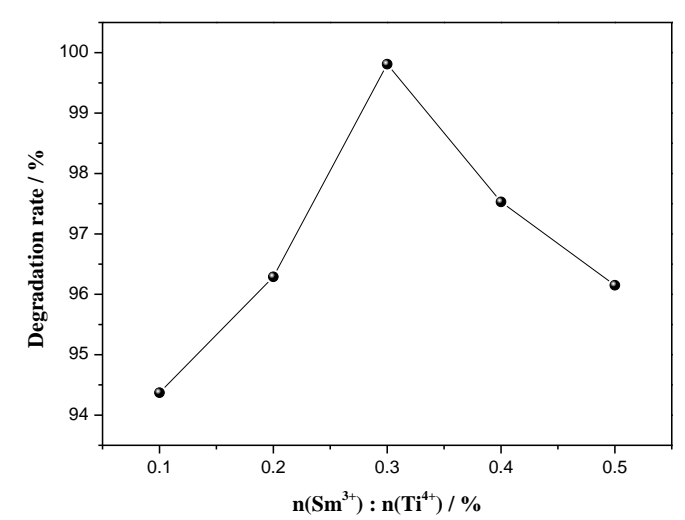

Figure 1. The influence of $\mathrm{n}(\mathrm{Sm} 3+) / \mathrm{n}(\mathrm{Ti} 4+)$ mole ratio on photocatalytic activity of $\mathrm{TiO} 2-\mathrm{Sm}$

2) Microwave-Ultrasonic enhanced photocatalytic activity

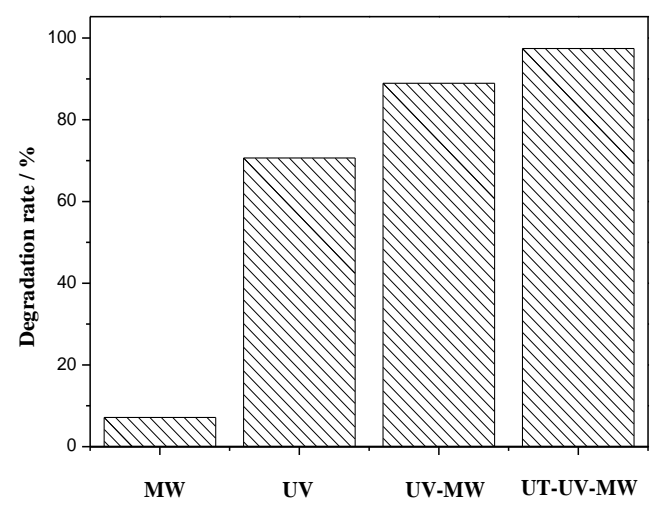

Figure 2. Degradation rate of methyl orange for TiO2-Sm under four conditions

Fig. 2 was effect of the four degradation conditions on the photocatalytic activity of TiO2-Sm for the degradation time of $15 \mathrm{~min}$. The results were that the degradation rate of $\mathrm{TiO} 2-\mathrm{Sm}$ was $7.16 \%$, $69.76 \%, 88.45 \%$ and $97.44 \%$ under MW, UV, UV-MW and UV-MW-UT. And more under different degradation conditions the degradation rate of $\mathrm{TiO} 2-\mathrm{Sm}$ was always UV-MW-UT $>$ UV$M W>U V>M W$ in the same reaction time. This showed that the effect of microwave radiation and ultrasonic vibration could enhance the photocatalytic degradation of organic pollutants by $\mathrm{TiO} 2-\mathrm{Sm}$ catalyst under UV irradiation. The possible reason was that: first, microwave radiation could make the surface of the TiO2-Sm catalyst produced more hydroxyl radicals, which could inhibit the photogenerated electron hole recombination $[12,13]$. Second, high frequency signal emitted by the ultrasonic vibration could effectively make the TiO2-Sm catalyst uniformly dispersed in the organic pollutants, which the catalyst and organic pollutants increased contact area. Third, ultraviolet irradiation, microwave radiation and ultrasonic vibration together in the reaction system produced additive effect, so as to improve the photocatalytic degradation activity. 


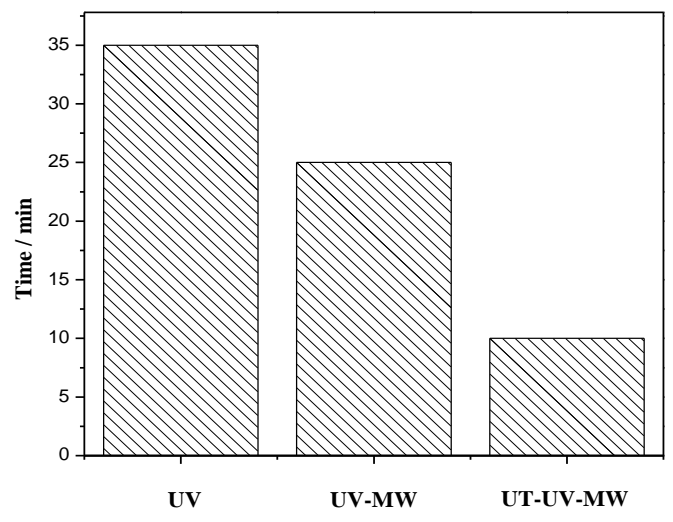

Figure 3. Relationship between reaction conditions and time

When the degradation rate of $\mathrm{TiO} 2-\mathrm{Sm}$ was greater than $90 \%$ under the UV, UV-MW and UTUV-MW reaction conditions, the relationship between reaction conditions and time was shown in Fig. 3. The result was that the degradation time was significantly $35 \mathrm{~min}, 25 \mathrm{~min}$ and $10 \mathrm{~min}$ when the degradation reaction achieved the requirements of degradation. This further showed that ultraviolet irradiation, microwave irradiation and ultrasonic vibration could produce synergistic effect and the MW-UV-UT was more conducive to the organic pollutants degradation of TiO2-Sm. The photocatalytic degradation time had shorten, which it improved the efficiency of photocatalytic reaction.

3) Stability of $\mathrm{TiO} 2-\mathrm{Sm}$ photocatalyst

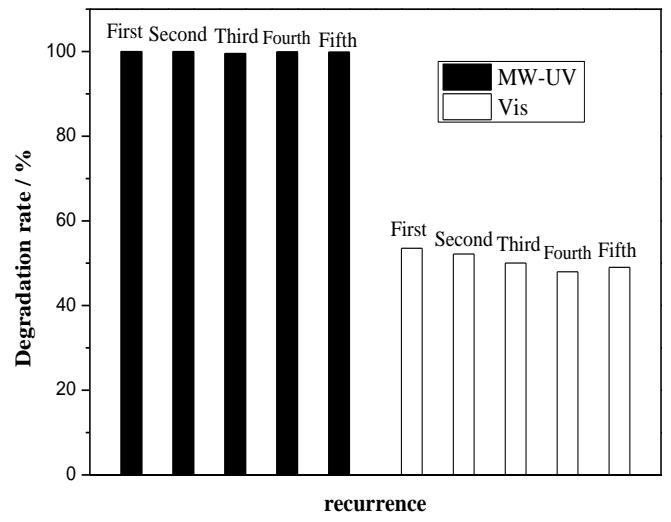

Figure 4. Results of TiO2-Sm catalyst stability test at MW-UV or visible light

The photocatalytic activity of $\mathrm{TiO} 2-\mathrm{Sm}$ was repeated degradation of methyl orange under the condition of MW-UV and visible light for each time being $55 \mathrm{~min}$ and $1 \mathrm{~h}$. The absorbance of the first reaction sample was measured and the degradation rate was calculated. The supernatant was removed and repeated washing, then drying to second reaction samples. The experiment was repeated 5 times in order to evaluate the stability of TiO2-Sm as shown in Fig. 4. The degradation rate was respectly $99.83 \%$ and $49.02 \%$ after repeated 5 tests. The degradation rate was nearly same as the first degradation rate $100 \%$ and $53.54 \%$. This result indicated that the $\mathrm{TiO} 2-\mathrm{Sm}$ catalyst had good heating stability.

SEM analysis. Fig. 5 was SEM diagram of the $0.3 \% \mathrm{TiO} 2-\mathrm{Sm}$ catalyst. The result showed that TiO2-Sm catalyst particles were rough surface with a plush shell and a small degree of aggregation of nano spheres. And more the TiO2-Sm catalyst could fully contact with the organic pollutants of 
methyl orange, so it was better to photocatalytic degradation and could improve its photocatalytic activity.

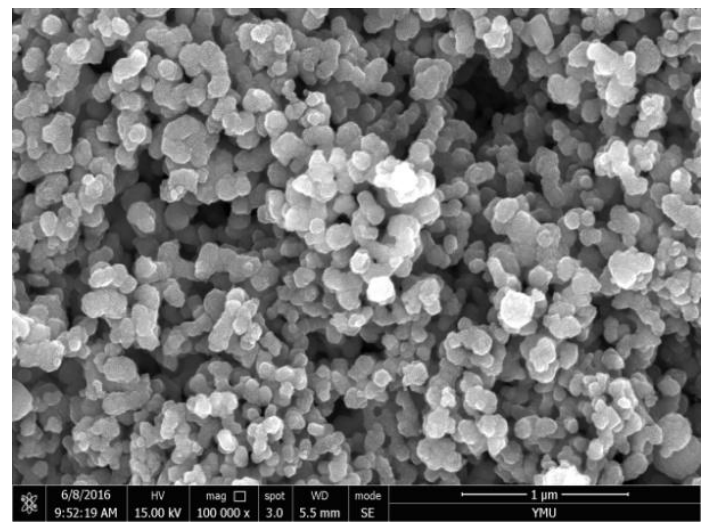

Figure 5. The SEM diagram of $0.3 \% \mathrm{TiO} 2-\mathrm{Sm}$

TG-DSC-DTG Analysis. Fig. 6 and Fig. 7 were respectly TG-DSC-DTG curves of pure $\mathrm{TiO}_{2}$ and $\mathrm{TiO}_{2}$-Sm prepared under the optimum preparation conditions. The result from Figure 6 showed that the curve could be divided into 3 stages. The first stage, the TG curve of $0 \sim 100^{\circ} \mathrm{C}$ range of weight loss was most serious, DSC curve had a peak temperature of $60^{\circ} \mathrm{C}$ strong exothermic peak which was of low boiling point organic matter decomposition and ethanol and water molecules caused by evaporation. Second order section, $100^{\circ} \mathrm{C} \sim 350^{\circ} \mathrm{C}$ range of weight loss had less, DSC curve had a peak temperature of $240^{\circ} \mathrm{C}$ strong endothermic peak which was the precursor of organic matter decomposition caused by combustion or tetrabutyl titanate. Third stages, weight loss was not obvious after $350^{\circ} \mathrm{C}$, the DSC curve was exothermic phenomenon, turning at a temperature of $390^{\circ} \mathrm{C}, 721^{\circ} \mathrm{C}$ and $850^{\circ} \mathrm{C}$ respectively, of which $390^{\circ} \mathrm{C} \sim 721^{\circ} \mathrm{C}$ was $\mathrm{TiO}_{2}$ from amorphous anatase transition anatase temperature, $721^{\circ} \mathrm{C} \sim 850^{\circ} \mathrm{C}$ for grain size and crystal growth of anatase phase into rutile the temperature, and $850^{\circ} \mathrm{C} \sim 1000^{\circ} \mathrm{C}$ was due to a large number of anatase growth for rutile and rutile grain size. Fig. 7 for the peak reason was similar; the $\mathrm{TG}$ curve of the $\mathrm{TiO}_{2}$-Sm could also be divided into 3 stages: weight loss at $0{ }^{\circ} \mathrm{C} \sim 100{ }^{\circ} \mathrm{C}$ and at $100{ }^{\circ} \mathrm{C} \sim 425{ }^{\circ} \mathrm{C}$, but weight loss was not obvious at $425{ }^{\circ} \mathrm{C}$. The basic weight loss after $425{ }^{\circ} \mathrm{C}$ showed an exothermic phenomenon of $\mathrm{TiO}_{2}-\mathrm{Sm}$ crystal from amorphous to anatase. Exothermic phenomenon was not obvious at $685{ }^{\circ} \mathrm{C} \sim 900{ }^{\circ} \mathrm{C}$, which was to the growth of anatase grains and change anatase to rutile.

The result of the heat release of $\mathrm{TiO}_{2}-\mathrm{Sm}$ after $375{ }^{\circ} \mathrm{C}$ being relatively flat and the amorphous anatase and rutile of transition temperature increased could be gained from the TG-DSC-DTG curve between $\mathrm{TiO}_{2}$ and $\mathrm{TiO}_{2}-\mathrm{Sm}$. This indicated that the $\mathrm{Sm}$ doped could broaden from amorphous to anatase temperature range, while inhibited the anatase structure into rutile structure.

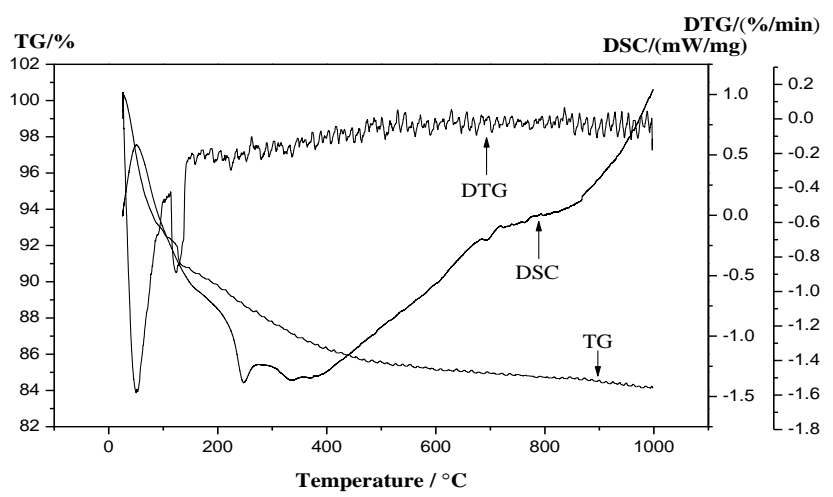

Figure 6. TG-DSC-DTG curve of TiO2 


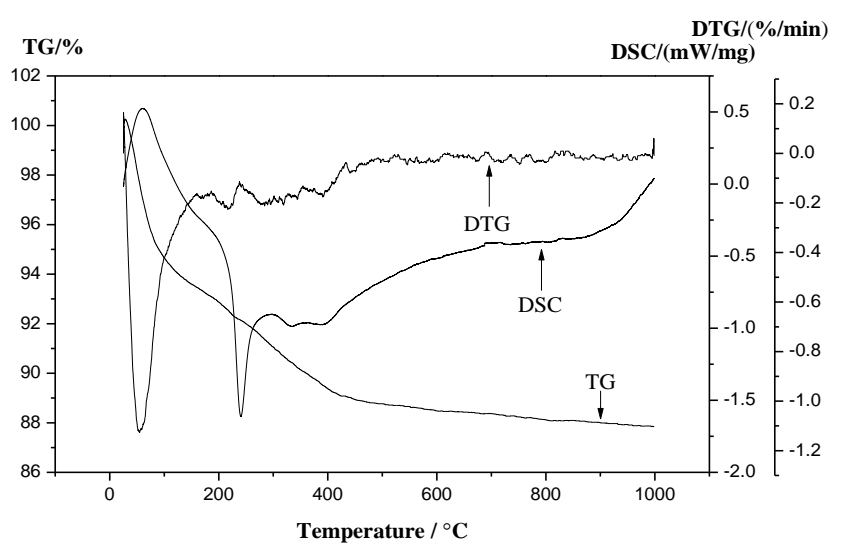

Figure 7. TG-DSC-DTG curve of TiO2-Sm

Infrared Spectrum Analysis. Fig. 8 was the infrared spectra of $\mathrm{TiO}_{2}$ and $\mathrm{TiO}_{2}-\mathrm{Sm}$ photocatalysts. The peak intensity and area indicated the strength of various groups, and the hydroxyl radical on the surface of the catalyst could improve the photocatalytic activity of the catalyst. For peak intensity, peak wide and the hydroxyl radical content, $\mathrm{TiO}_{2}-\mathrm{Sm}$ was significantly higher than pure $\mathrm{TiO}_{2}$. This showed that $\mathrm{Sm}$ doped produced more hydroxyl groups and enhanced the photocatalytic activity of $\mathrm{TiO}_{2}-\mathrm{Sm}$, which improved the efficiency of the degradation of organic pollutants, this studied consistent with the results of Horikoshi et $\mathrm{al}^{[14]}$. Moreover, there was no new absorption peaks in the spectrum, which maight be $\mathrm{Sm}$ did not affect $\mathrm{TiO}_{2}$ photocatalyst in various key. Doped amount was too small to no correlation peak with Sm.

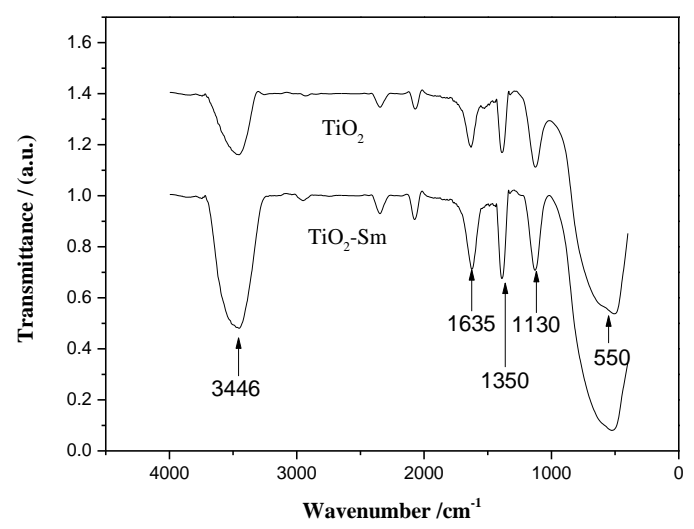

Figure 8. IR spectra of $\mathrm{TiO} 2$ and $\mathrm{TiO} 2-\mathrm{Sm}$

$$
\text { (A:TiO2 B: TiO2-Sm) }
$$

UV-Vis Analysis. The UV visible spectrum could characterize the light absorption ability. The characterization of results of pure $\mathrm{TiO}_{2}$ and $\mathrm{Sm}$ doped $\mathrm{TiO}_{2}$ powder were shown in Fig. 9. $\mathrm{TiO}_{2}-\mathrm{Sm}$ and pure $\mathrm{TiO}_{2}$ had the wide strong absorption in the range of 200-800 nm, but the absorption intensity of $\mathrm{TiO}_{2}-\mathrm{Sm}$ was higher than that pure $\mathrm{TiO}_{2}$. Due to the $\mathrm{Sm}$ doped, the optical absorption band was red shift from pure $\mathrm{TiO}_{2}(390 \mathrm{~nm})$ to $\mathrm{TiO}_{2}-\mathrm{Sm}(435 \mathrm{~nm})$. This showed that $\mathrm{Sm}$ doped could broaden the range of absorption in the ultraviolet and visible light, which made the photocatalytic activity of doped catalysts significantly increased ${ }^{[15]}$. 


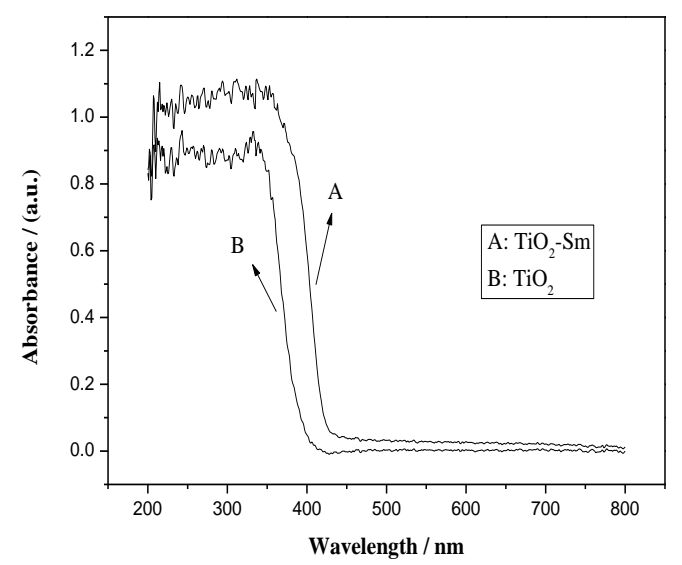

Figure 9. Uv-Vis spectra of pure $\mathrm{TiO} 2$ and $\mathrm{TiO} 2-\mathrm{Sm}$ powders (A: $\mathrm{TiO} 2-\mathrm{Sm}$ B: pure $\mathrm{TiO} 2$ )

\section{Conclusion}

(1) The optimum conditions of $\mathrm{TiO} 2-\mathrm{Sm}$ photocatalyst prepared by microwave hydrothermal method were as follows: $\mathrm{n}(\mathrm{Sm} 3+) / \mathrm{n}(\mathrm{Ti} 4+)=0.003$, microwave reaction power $450 \mathrm{~W}$, reaction temperature $170^{\circ} \mathrm{C}$, reaction time $3 \mathrm{~h}$, calcination temperature $700^{\circ} \mathrm{C}$ and calcination time $3 \mathrm{~h}$.

(2) By optimizing the reaction conditions to prepare $\mathrm{TiO} 2-\mathrm{Sm}$ had high photocatalytic activity. The degradation rate was $11.51 \%, 96.83 \%, 99.5 \%$ and $100 \%$ respectively by MW, UV, UV-MW and UV-MW-UT for 35 min degradation.

(3) The results from $\mathrm{TiO} 2-\mathrm{Sm}$ characterization showed that $\mathrm{Sm}$ doped had great influence on the crystal formation which widen the temperature range of catalyst from amorphous to anatase and suppressing the anatase structure into rutile structure. Moreover Sm doped produced more hydroxyl groups and could also widen the absorb range of ultraviolet and visible light which caused the enhancement of the photocatalytic activity of TiO2-Sm.

\section{References}

[1] Cui Yumin, Shan Dejie, Zhu Yiren. Studies on photocatalytic oxidation of I- over TiO2 thin film. J. Chinese Journal of Inorganic Chemistry, 2001, 17(3):401-406.

[2] Cui Yumin. Photocatalytic degradat of pollutant from river by $\mathrm{TiO} 2$ as photocatalyst. J. Journal of Henan University of Science and Technology (Natural Science), 2003, 24(1):94-97.

[3] Cui Yumin, Fan shaohua. Present advance and tendency of photocatalytic process in sewage disposal. J. Journal of LuoYang Institute of Technology (Natural Science), 2002, 23(2):85-89.

[4] Wang Yan, Zhao Hui, Zhang Jiwei and etal. Research progress of visible light Photocatalysis of nitrogen-doped TiO2. J. Henan Chemical Industry, 2008, 25(5):5-9

[5] Xu Anwu, Gao Yuan, Liu Hanqin. The preparation, characterization, and their photocatalytic activities of rare-earth-doped TiO2 nanoparticles. J. Journal of Catalysis, 2002, 207(2): 151-157.

[6] Zhang Mei, Xu Yaling, Bi Xianjun. Study on microwave-hydrothermal and sol-gel method synthesis of Er-doped $\mathrm{TiO} 2$ photocatalyst and its photo-chemistry performance. C. Advances in Computer Science Research-Management, Computer and Education Informatization, Paris: Atlantis Press, 2016:1204-1207. 
[7] Li Manwan, Zhang Mei, Bi Xianjun. Preparation of Ce-doped TiO2 photocatalyst and its microwave enhanced photocatalytic activity. J. Industrial Catalysis, 2015, 23(12):986-990.

[8] Li Manwan, Zhang Mei, Bi Xianjun. Preparation of Y-Ce doped with $\mathrm{TiO} 2$ photocatalyst in microwave ionic liqids and microwave enhanced photocatalytic activity. C. Advances in Computer Science Research-Management, Computer and Education Informatization, Paris: Atlantis Press, 2015:532-535.

[9] Zhang Mei, Lu Yuejie, Bi Xianjun. Preparation of Yb-Er Doped with TiO2 photocatalyst in microwave ionic liqids and microwave enhanced photocatalytic activity. C. Advances in Computer Science Research-Management, Computer and Education Informatization, Paris: Atlantis Press, 2016:1362-1365.

[10]Zielinska A, Kowalska E, Sobczak J W and etal. Silver-doped TiO2 prepared by microemulsion method: Surface properties, bio- and photoacitivity. J. Separation \& Purification Technology, 2010, 72(3):309-318.

[11] Sobana N, Muruganadham M, Swaminathan M. Nano-Ag particles doped TiO2 for efficient photodegradation of Direct azo dyes. J. Journal of Molecular Catalysis A Chemical, 2006, 258(1-2):124-132.

[12]Zhai Chunyang, Zhou Weiqiang, Xu Jingkun and etal. Preparation and properties of magnetic photocatalyst: TiO2-Fe3O4 loaded on active carbon. J. Chemical Research, 2009, 20(02): 83-86.

[13] Li Yu, Han Zhaorang, Zhai Yongai and etal. Preparation and characterization of $\mathrm{TiO} 2 / \mathrm{SiO} 2$ composite materials. J. Chemical Research, 2007, 18(01): 19-22.

[14]Horikoshi S, Hidaka H. Environmental remediation by an integrated microwave/UV illumination method microwave-assisted degradation of rhodamine $\mathrm{B}$ dye in aqueous $\mathrm{TiO} 2$ dispersions. J. Environmental Science and Technology, 2002, 36:1357-1366.

[15]Liu jun. Preparation of TiO2 Photocatalysts and Their Application in degradation of formic acid and methyl orange. D. Chengdu: Sichuan University, 2002. 